\title{
HOMOGENIZATION OF HIGHLY OSCILLATING BOUNDARIES AND REDUCTION OF DIMENSION FOR A MONOTONE PROBLEM
}

\author{
Dominique Blanchard ${ }^{1}$ and Antonio Gaudiello ${ }^{2}$
}

\begin{abstract}
We investigate the asymptotic behaviour, as $\varepsilon \rightarrow 0$, of a class of monotone nonlinear Neumann problems, with growth $p-1(p \in] 1,+\infty[)$, on a bounded multidomain $\Omega_{\varepsilon} \subset \mathbb{R}^{N}(N \geq 2)$. The multidomain $\Omega_{\varepsilon}$ is composed of two domains. The first one is a plate which becomes asymptotically flat, with thickness $h_{\varepsilon}$ in the $x_{N}$ direction, as $\varepsilon \rightarrow 0$. The second one is a "forest" of cylinders distributed with $\varepsilon$-periodicity in the first $N-1$ directions on the upper side of the plate. Each cylinder has a small cross section of size $\varepsilon$ and fixed height (for the case $N=3$, see the figure). We identify the limit problem, under the assumption: $\lim _{\varepsilon \rightarrow 0} \frac{\varepsilon^{p}}{h_{\varepsilon}}=0$. After rescaling the equation, with respect to $h_{\varepsilon}$, on the plate, we prove that, in the limit domain corresponding to the "forest" of cylinders, the limit problem identifies with a diffusion operator with respect to $x_{N}$, coupled with an algebraic system. Moreover, the limit solution is independent of $x_{N}$ in the rescaled plate and meets a Dirichlet transmission condition between the limit domain of the "forest" of cylinders and the upper boundary of the plate.
\end{abstract}

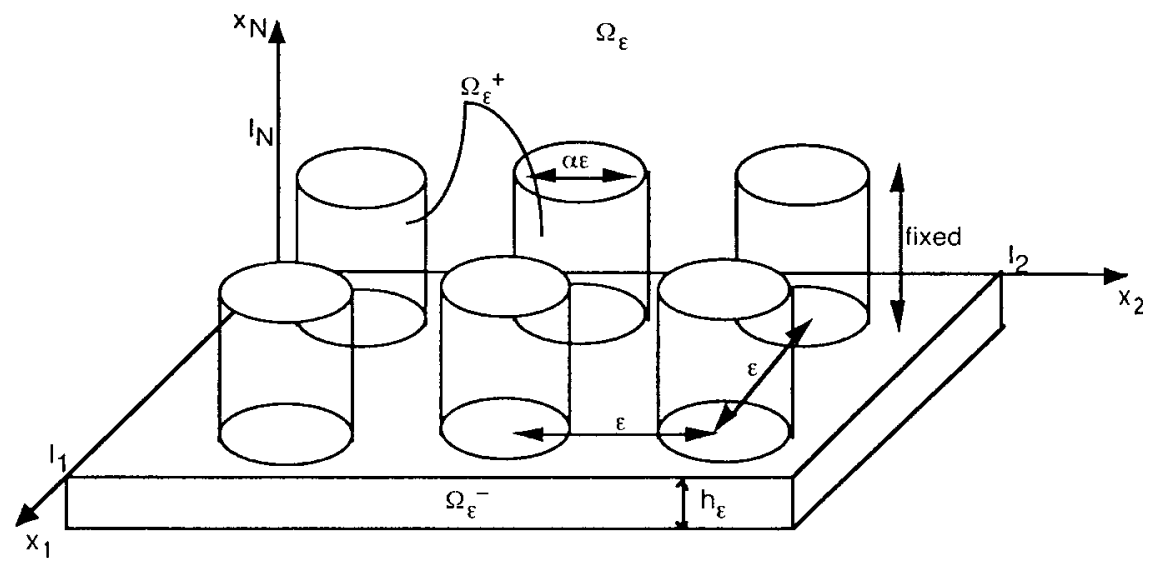

Mathematics Subject Classification. 35B27, 35J60.

Received November 22, 2002. Revised February 7, 2003.

Keywords and phrases. Homogenization, oscillating boundaries, multidomain, monotone problem.

${ }^{1}$ Université de Rouen, UMR 6085, 76821 Mont-Saint-Aignan Cedex, France, and Laboratoire d'Analyse Numérique, Université P. et M. Curie, Case Courrier 187, 75252 Paris Cedex 05, France; e-mail: blanchar@ann.jussieu.fr

2 Università degli Studi di Cassino, Dipartimento di Automazione, Elettromagnetismo, Ingegneria dell'Informazione e Matematica Industriale, via G. di Biasio 43, 03043 Cassino (FR), Italy; e-mail: gaudiell@unina.it 


\section{Motivation AND MAin Result}

Let $\left.N \geq 2, l_{1}, \cdots, l_{N-1}, l_{N} \in\right] 0,+\infty\left[, \omega\right.$ be an open smooth subset of $\mathbb{R}^{N-1}$ such that $\left.\omega \subset \subset\right] 0,1\left[{ }^{N-1}\right.$, and let $\{\varepsilon\},\left\{h_{\varepsilon}\right\}_{\varepsilon}$ be two sequences of strictly positive numbers converging to 0 . For every $\varepsilon$, let us consider the multidomain in $\mathbb{R}^{N}$ (for the case $N=3$, see the figure):

$$
\Omega_{\varepsilon}=\Omega_{\varepsilon}^{-} \cup \Omega_{\varepsilon}^{+}
$$

where $\Omega_{\varepsilon}^{-}$is a plate with constant cross section and small height $h_{\varepsilon}$ :

$$
\left.\Omega_{\varepsilon}^{-}=\right] 0, l_{1}[\times \cdots \times] 0, l_{N-1}[\times]-h_{\varepsilon}, 0[,
$$

and $\Omega_{\varepsilon}^{+}$is a "forest" of cylinders with small cross section $\varepsilon \omega$, constant height $l_{N}$ and distributed with $\varepsilon$-periodicity in the first $N-1$ directions $x_{1}, \cdots, x_{N-1}$ on the upper side of $\Omega_{\varepsilon}^{-}$:

$$
\Omega_{\varepsilon}^{+}=\bigcup_{k \in J_{\varepsilon}}(\varepsilon \omega+\varepsilon k) \times\left[0, l_{N}[\right.
$$

where

$$
J_{\varepsilon}=\left\{k \in \mathbb{N}^{N-1}: \varepsilon \omega+\varepsilon k \subset \subset\right] 0, l_{1}[\times \cdots \times] 0, l_{N-1}[\} .
$$

Let us observe that the volume of $\Omega_{\varepsilon}^{+}$does not vanish as $\varepsilon \rightarrow 0$, precisely:

$$
\chi_{\Omega_{\varepsilon}^{+}} \rightarrow|\omega| \quad \text { in } L^{\infty}\left(\Omega^{+}\right) \text {weak } *
$$

where $\chi_{\Omega_{\varepsilon}^{+}}$denotes the characteristic function of $\Omega_{\varepsilon}^{+},|\omega|$ denotes the $(N-1)$-dimensional Lebesgue measure of $\omega$ and $\Omega^{+}$is the "smallest" parallelepiped containing the interior of $\Omega_{\varepsilon}^{+}$for every $\varepsilon$ :

$$
\left.\Omega^{+}=\right] 0, l_{1}[\times \cdots \times] 0, l_{N-1}[\times] 0, l_{N}[.
$$

This paper arises from the interest of studying the asymptotic behaviour, as $\varepsilon \rightarrow 0$, of the following Neumann problem:

$$
\left\{\begin{array}{l}
-\operatorname{div}\left(a\left(D U_{\varepsilon}\right)\right)+\left|U_{\varepsilon}\right|^{p-2} U_{\varepsilon}=F_{\varepsilon} \quad \text { in } \Omega_{\varepsilon}, \\
a\left(D U_{\varepsilon}\right) \cdot \nu=0 \text { on } \partial \Omega_{\varepsilon},
\end{array}\right.
$$

where $\nu$ denotes the exterior unit normal to $\left.\Omega_{\varepsilon}, p \in\right] 1,+\infty\left[, F_{\varepsilon} \in L^{\frac{p}{p-1}}\left(\Omega^{+} \cup \Omega_{\varepsilon}^{-}\right)\right.$and

$$
a=\left(a_{1}, \cdots, a_{N}\right): \mathbb{R}^{N} \rightarrow \mathbb{R}^{N} \text { is a monotone continuous function }
$$

satisfying the usual growth conditions:

$$
\begin{gathered}
\exists \alpha \in] 0,+\infty\left[: \alpha|\xi|^{p} \leq a(\xi) \xi \quad \forall \xi \in \mathbb{R}^{N},\right. \\
\exists \beta, \gamma \in] 0,+\infty\left[:|a(\xi)| \leq \beta+\gamma|\xi|^{p-1} \quad \forall \xi \in \mathbb{R}^{N} .\right.
\end{gathered}
$$

It is well known (see [18]) that problem (1.3) admits a unique weak solution $U_{\varepsilon} \in W^{1, p}\left(\Omega_{\varepsilon}\right)$. To study the asymptotic behaviour of $\left\{U_{\varepsilon}\right\}_{\varepsilon}$, as $\varepsilon \rightarrow 0$, we introduce the classical transformation mapping $\Omega_{\varepsilon}^{-}$onto the fixed domain

$$
\left.\Omega^{-}=\right] 0, l_{1}[\times \cdots \times] 0, l_{N-1}[\times]-1,0[
$$

(compare, for instance $[9,14,16]$ and $[17])$ and we set, for every $\varepsilon$,

$$
u_{\varepsilon}(x)=\left\{\begin{array}{l}
U_{\varepsilon}(x) \quad x \text { a.e. in } \Omega_{\varepsilon}^{+}, \\
U_{\varepsilon}\left(x^{\prime}, h_{\varepsilon} x_{N}\right) \quad x=\left(x^{\prime}, x_{N}\right) \text { a.e. in } \Omega^{-} .
\end{array}\right.
$$


Obviously, $u_{\varepsilon}$ is the unique solution of the following problem:

$$
\left\{\begin{array}{l}
\int_{\Omega_{\varepsilon}^{+}} a\left(D u_{\varepsilon}\right) D v+\left|u_{\varepsilon}\right|^{p-2} u_{\varepsilon} v \mathrm{~d} x \\
+h_{\varepsilon} \int_{\Omega^{-}} a\left(D_{x^{\prime}} u_{\varepsilon}, \frac{1}{h_{\varepsilon}} \frac{\partial u_{\varepsilon}}{\partial x_{N}}\right)\left(D_{x^{\prime}} v, \frac{1}{h_{\varepsilon}} \frac{\partial v}{\partial x_{N}}\right)+\left|u_{\varepsilon}\right|^{p-2} u_{\varepsilon} v \mathrm{~d} x \\
=\int_{\Omega_{\varepsilon}^{+}} f_{\varepsilon} v \mathrm{~d} x+h_{\varepsilon} \int_{\Omega^{-}} f_{\varepsilon} v \mathrm{~d} x \quad \forall v \in W^{1, p}\left(\Omega_{\varepsilon}^{+} \cup \Omega^{-}\right) \\
u_{\varepsilon} \in W^{1, p}\left(\Omega_{\varepsilon}^{+} \cup \Omega^{-}\right)
\end{array}\right.
$$

where $x=\left(x_{1}, \cdots, x_{N-1}, x_{N}\right)=\left(x^{\prime}, x_{N}\right) \in \mathbb{R}^{N}, D_{x^{\prime}}=\left(\frac{\partial}{\partial x_{1}}, \cdots, \frac{\partial}{\partial x_{N-1}}\right)$ and

$$
f_{\varepsilon}(x)=\left\{\begin{array}{l}
F_{\varepsilon}(x) \quad x \text { a.e. in } \Omega^{+}, \\
F_{\varepsilon}\left(x^{\prime}, h_{\varepsilon} x_{N}\right) \quad x=\left(x^{\prime}, x_{N}\right) \text { a.e. in } \Omega^{-} .
\end{array}\right.
$$

Then, we study the asymptotic behaviour, as $\varepsilon \rightarrow 0$, of problem (1.7), under the following supplementary assumptions:

$$
\begin{gathered}
\lim _{\varepsilon \rightarrow 0} \frac{\varepsilon^{p}}{h_{\varepsilon}}=0 \\
\left\{\begin{array}{l}
f_{\varepsilon} \rightarrow f \text { strongly in } L^{\frac{p}{p-1}}\left(\Omega^{+}\right), \\
\exists c \in] 0,+\infty\left[:\left\|f_{\varepsilon}\right\|_{L^{\frac{p}{p-1}}\left(\Omega^{-}\right)} \leq c, \quad \forall \varepsilon .\right.
\end{array}\right.
\end{gathered}
$$

In terms of the sequence $\left\{F_{\varepsilon}\right\}_{\varepsilon},(1.10)$ means that $\left\{F_{\varepsilon}\right\}_{\varepsilon}$ converges strongly in $L^{\frac{p}{p-1}}\left(\Omega^{+}\right)$and $\left\|F_{\varepsilon}\right\|_{L^{\frac{p}{p-1}}\left(\Omega_{\varepsilon}^{-}\right)}$ $\leq c h_{\varepsilon}^{\frac{p-1}{p}}$.

To describe the limit problem, we introduce the space

$$
V^{p}\left(\Omega^{+}\right)=\left\{v \in L^{p}\left(\Omega^{+}\right): \frac{\partial v}{\partial x_{N}} \in L^{p}\left(\Omega^{+}\right)\right\},
$$

and we recall that functions of $V^{p}\left(\Omega^{+}\right)$admit a trace on $\Sigma$ :

$$
\Sigma=] 0, l_{1}[\times \cdots \times] 0, l_{N-1}[\times\{0\} .
$$

In the sequel, $\widetilde{v}$ or $[v]^{\sim}$ denotes the zero-extension to $\Omega^{+}$of any (vector) function $v$ defined on a subset of $\Omega^{+}$.

The main result of this paper is the following one:

Theorem 1.1. Let $u_{\varepsilon}$ be the unique solution of problem (1.7) under assumptions (1.4-1.6, 1.9, 1.10). Let |w| be the constant given by (1.2) and let $V^{p}\left(\Omega^{+}\right)$be the space defined in (1.11).

Then, there exists $u \in V^{p}\left(\Omega^{+}\right)$such that

$$
\begin{gathered}
\widetilde{u_{\varepsilon}} \rightarrow|\omega| u, \quad \frac{\partial \widetilde{u_{\varepsilon}}}{\partial x_{N}} \rightarrow|\omega| \frac{\partial u}{\partial x_{N}} \quad \text { weakly in } L^{p}\left(\Omega^{+}\right), \\
u_{\varepsilon} \rightarrow u_{\mid \Sigma} \text { weakly in } L^{p}\left(\Omega^{-}\right), \\
\frac{\partial u_{\varepsilon}}{\partial x_{N}} \rightarrow 0 \text { strongly in } L^{p}\left(\Omega^{-}\right),
\end{gathered}
$$


as $\varepsilon \rightarrow 0$, where $u_{\mid \Sigma}$ denotes the function independent of $x_{N}$ which is equal, on $\Sigma$, to the trace of $u$. Moreover, there exist a subsequence of $\{\varepsilon\}$, still denoted by $\{\varepsilon\}$, and $\left(d_{1}, \cdots, d_{N-1}\right) \in\left(L^{p}\left(\Omega^{+}\right)\right)^{N-1}$, depending possibly on the selected subsequence, such that

$$
\widetilde{\partial u_{\varepsilon}} \overrightarrow{\partial x_{i}} \rightarrow d_{i} \quad \text { weakly in } L^{p}\left(\Omega^{+}\right) \quad \forall i \in\{1, \cdots, N-1\}
$$

as $\varepsilon \rightarrow 0$, and $\left(u, d_{1}, \cdots, d_{N-1}\right)$ is a weak solution of the following problem:

$$
\left\{\begin{array}{l}
-\frac{\partial}{\partial x_{N}} a_{N}\left(\frac{d_{1}}{|\omega|}, \cdots, \frac{d_{N-1}}{|\omega|}, \frac{\partial u}{\partial x_{N}}\right)+|u|^{p-2} u=f \quad \text { in } \Omega^{+}, \\
a_{N}\left(\frac{d_{1}}{|\omega|}, \cdots, \frac{d_{N-1}}{|\omega|}, \frac{\partial u}{\partial x_{N}}\right)=0 \quad \text { on the lower and upper boundary of } \Omega^{+}, \\
a_{i}\left(\frac{d_{1}}{|\omega|}, \cdots, \frac{d_{N-1}}{|\omega|}, \frac{\partial u}{\partial x_{N}}\right)=0 \quad \text { a.e. in } \Omega^{+}, \quad \forall i \in\{1, \cdots, N-1\} .
\end{array}\right.
$$

The function $u \in V^{p}\left(\Omega^{+}\right)$satisfying problem (1.16) is unique. Furthermore, the energies converge in the sense that:

$$
\begin{aligned}
\lim _{\varepsilon \rightarrow 0}\left(\int_{\Omega_{\varepsilon}^{+}} a\left(D u_{\varepsilon}\right) D u_{\varepsilon}+\left|u_{\varepsilon}\right|^{p} \mathrm{~d} x\right. & \left.+h_{\varepsilon} \int_{\Omega^{-}} a\left(D_{x^{\prime}} u_{\varepsilon}, \frac{1}{h_{\varepsilon}} \frac{\partial u_{\varepsilon}}{\partial x_{N}}\right)\left(D_{x^{\prime}} u_{\varepsilon}, \frac{1}{h_{\varepsilon}} \frac{\partial u_{\varepsilon}}{\partial x_{N}}\right)+\left|u_{\varepsilon}\right|^{p} \mathrm{~d} x\right) \\
& =|\omega| \int_{\Omega^{+}} a_{N}\left(\frac{d_{1}}{|\omega|}, \cdots, \frac{d_{n-1}}{|\omega|}, \frac{\partial u}{\partial x_{N}}\right) \frac{\partial u}{\partial x_{N}}+|u|^{p} \mathrm{~d} x=|\omega| \int_{\Omega^{+}} f u \mathrm{~d} x
\end{aligned}
$$

If a is strictly monotone, problem (1.16) admits a unique solution $\left(u, d_{1}, \cdots, d_{N-1}\right) \in V^{p}\left(\Omega^{+}\right) \times\left(L^{p}\left(\Omega^{+}\right)\right)^{N-1}$ and, consequently, convergence (1.15) holds true for the whole sequence $\left\{u_{\varepsilon}\right\}_{\varepsilon}$.

We point out that the limit problem, in the limit domain corresponding to the "forest" of cylinders, identifies with a diffusion operator with respect to $x_{N}$ coupled with an algebraic system for the limit fluxes. In particular, if $a(\xi)=|\xi|^{p-2} \xi$, with $p \in\left[2,+\infty\right.$, it results $d_{1}=\cdots=d_{N-1}=0$ a.e. in $\Omega^{+}$.

As far as the asymptotic behaviour, as $\varepsilon \rightarrow 0$, of the solution $U_{\varepsilon}$ of problem (1.3) is concerned, Theorem 1.1 leads immediately to the following result $\left(\left|\Omega_{\varepsilon}^{-}\right|\right.$and $|\Sigma|$ denote the $N$-dimensional Lebesgue measure of $\Omega_{\varepsilon}^{-}$and the $(N-1)$-dimensional Lebesgue measure of $\Sigma$, respectively):

Corollary 1.2. Let $U_{\varepsilon}$ be the unique solution of problem (1.3). Then, under the assumptions of Theorem 1.1 with $f_{\varepsilon}$ defined by (1.8), it results

$$
\begin{gathered}
\widetilde{U_{\varepsilon}} \rightarrow|\omega| u, \quad \frac{\partial \widetilde{U_{\varepsilon}}}{\partial x_{N}} \rightarrow|\omega| \frac{\partial u}{\partial x_{N}} \quad \text { weakly in } L^{p}\left(\Omega^{+}\right) \\
\frac{\widetilde{\partial U_{\varepsilon}}}{\partial x_{i}} \rightarrow d_{i} \quad \text { weakly in } L^{p}\left(\Omega^{+}\right) \quad \forall i \in\{1, \cdots, N-1\}, \text { up to a subsequence, } \\
\lim _{\varepsilon} \int_{\Omega_{\varepsilon}^{-}}\left|U_{\varepsilon}\right|^{p} \mathrm{~d} x=0 \\
\lim _{\varepsilon} \frac{1}{\left|\Omega_{\varepsilon}^{-}\right|} \int_{\Omega_{\varepsilon}^{-}} U_{\varepsilon} \mathrm{d} x=\frac{1}{|\Sigma|} \int_{\Sigma} u \mathrm{~d} x^{\prime}
\end{gathered}
$$

as $\varepsilon \rightarrow 0$, and $\left(u, d_{1}, \cdots, d_{N-1}\right) \in V^{p}\left(\Omega^{+}\right) \times\left(L^{p}\left(\Omega^{+}\right)\right)^{N-1}$ is a weak solution of problem (1.16). Moreover, the energies converge in the sense that:

$$
\lim _{\varepsilon \rightarrow 0} \int_{\Omega_{\varepsilon}} a\left(D U_{\varepsilon}\right) D U_{\varepsilon}+\left|U_{\varepsilon}\right|^{p} \mathrm{~d} x=|\omega| \int_{\Omega^{+}} a_{N}\left(\frac{d_{1}}{|\omega|}, \cdots, \frac{d_{n-1}}{|\omega|}, \frac{\partial u}{\partial x_{N}}\right) \frac{\partial u}{\partial x_{N}}+|u|^{p} \mathrm{~d} x .
$$


Remark 1.3. We point out that we need assumption (1.9) only to derive convergence (1.13), and consequently (1.18). The remaining part of Theorem 1.1 and of Corollary 1.2 holds true without assumption (1.9). In particular, the limit problem in $\Omega^{+}$can be obtained independently of the rate of convergence to zero of $h_{\varepsilon}$ with respect to $\varepsilon$. Assumption (1.9) is needed to describe the behaviour of the sequence $\left\{u_{\varepsilon}\right\}_{\varepsilon}$ in $\Omega^{-}$.

Remark 1.4. We point out that, under assumption (1.9), the results of Theorem 1.1 and Corollary 1.2 do not change if we replace assumption (1.10) by the weaker one

$$
\left\{\begin{array}{l}
f_{\varepsilon} \rightarrow f \text { strongly in } L^{\frac{p}{p-1}}\left(\Omega^{+}\right), \\
\exists c \in] 0,+\infty\left[:\left\|h_{\varepsilon}^{\frac{p-1}{p}} f_{\varepsilon}\right\|_{L^{\frac{p}{p-1}}\left(\Omega^{-}\right)} \leq c, \quad \forall \varepsilon\right.
\end{array}\right.
$$

that is $\left\{F_{\varepsilon}\right\}_{\varepsilon}$ converges strongly in $L^{\frac{p}{p-1}}\left(\Omega^{+}\right)$and $\left\|F_{\varepsilon}\right\|_{L^{\frac{p}{p-1}}\left(\Omega_{\varepsilon}^{-}\right)} \leq c$.

Also in the case where the rate of convergence of $h_{\varepsilon}$ is $\varepsilon^{p}$, under assumption (1.19), we obtain in $\Omega^{+}$the same limit problem as in Theorem 1.1.

If $h_{\varepsilon}=1$, the asymptotic behaviour of problem (1.3) (or (1.7)) has been studied by Blanchard et al. in [5]. Let us also recall a few references in the case where $h_{\varepsilon}=1$. For the linear problem, see [6] for homogeneous Neumann boundary condition, [12] for a non-homogeneous Neumann boundary condition, [13] for a transmission boundary condition, [20] for a spectral problem, and [3] and [19] for the construction of boundary layer correctors. Moreover, see [11] in the case of functionals with convex energies and [4] in the case of functionals with non-convex energies. Furthermore, see [15] for the Ginzburg-Landau equation with homogeneous Neumann boundary condition.

The asymptotic behaviour of the solution of problem (1.7) in $\Omega^{+}$is performed, with similar techniques than in [5], by making use of the method of oscillating test functions, introduced by Tartar in [22], combined with monotonicity arguments and density results (this is the object of Sect. 4). The originality of our paper consists in the study of the asymptotic behaviour of the solution of problem (1.7) in $\Omega^{-}$and in the characterization of the limit in $\Omega^{-}$in terms of the limit in $\Omega^{+}$(see (1.13)). The first task is to obtain a priori $L^{p}\left(\Omega^{-}\right)$-norm estimate for the solution $u_{\varepsilon}$, independently of $\varepsilon$. These estimates are not obvious since directly from equation (1.7) it follows only that $\left\|h_{\varepsilon}^{\frac{1}{p}} u_{\varepsilon}\right\|_{L^{p}\left(\Omega^{-}\right)} \leq c$. Via trace arguments (Friedrichs inequality), in Proposition 3.3 we are able to prove that $\left\|u_{\varepsilon}\right\|_{L^{p}\left(\Omega^{-}\right)} \leq c$. Consequently, by passing to a subsequence, $\left\{u_{\varepsilon}\right\}_{\varepsilon}$ converges to a function $w$ weakly in $L^{p}\left(\Omega^{-}\right)$and moreover, since $\left\|\frac{\partial u_{\varepsilon}}{\partial x_{N}}\right\|_{L^{p}\left(\Omega^{-}\right)} \leq c h_{\varepsilon}^{\frac{p-1}{p}}$, it follows that $w$ is independent of the last variable $x_{N}$. The second task is to identify $w$ as the trace on $\Sigma$ (the surface which separates $\Omega^{-}$and $\Omega^{+}$) of the solution $u$ of the limit problem in $\Omega^{+}$(see (1.13)). To this aim, we have to pass to the limit in the product of weak convergences: $\widetilde{u_{\varepsilon}}=u_{\varepsilon} \chi_{\Omega_{\varepsilon}^{+} \cap \Sigma}$ on $\Sigma$. In Proposition 3.4 we are able to solve this problem by making use of the two-scale convergence method introduced by Nguetseng in [21] and developed by Allaire in [1]. In Section 2 some preliminary results are recalled.

If the Neumann boundary condition in problem (1.3) is replaced by the homogeneous Dirichlet condition $U_{\varepsilon}=0$ on $\partial \Omega_{\varepsilon}$, it becomes an easier task to prove that $\widetilde{u_{\varepsilon}} \rightarrow 0$ weakly in $W^{1, p}\left(\Omega^{+}\right), u_{\varepsilon} \rightarrow 0$ strongly in $L^{p}\left(\Omega^{-}\right)$ (compare [3] and [6]). As far as this Dirichlet problem is concerned, the lower order term $\left|U_{\varepsilon}\right|^{p-2} U_{\varepsilon}$ may be removed in the whole analysis.

For the study of very rapidly oscillating boundaries we refer to $[2,4,7]$ and [8]. For the junction of a plate with a beam we refer to $[14,16]$ and $[17]$.

\section{Preliminary Results}

In this section, the main properties of the two-scale convergence method introduced by Nguetseng in [21] and developed by Allaire in [1] are recalled. Here, $Y=[0,1]^{N}(N \geq 1), 1<p<\infty, C_{\mathrm{per}}^{\infty}(Y)$ denotes the space of infinitely differentiable functions in $\mathbb{R}^{N}$ that are periodic of period $Y$ and $W_{\text {per }}^{1, p}(Y)$ is the completion of $C_{\text {per }}^{\infty}(Y)$ for the norm of $W^{1, p}(Y)$. 
Definition 2.1. Let $A$ be an open subset of $\mathbb{R}^{N}$. A sequence $\left\{v_{\varepsilon}\right\}_{\varepsilon} \subset L^{p}(A)$ is said to "two-scale converge" to a limit $v \in L^{p}(A \times Y)$ if, for any function $\psi$ in $D\left(A, C_{\mathrm{per}}^{\infty}(Y)\right)$, it results

$$
\lim _{\varepsilon \rightarrow 0} \int_{A} v_{\varepsilon}(x) \psi\left(x, \frac{x}{\varepsilon}\right) \mathrm{d} x=\int_{A \times Y} v(x, y) \psi(x, y) \mathrm{d} x \mathrm{~d} y .
$$

See [1] for the proof of the following result:

Proposition 2.2. Let $A$ be an open subset of $\mathbb{R}^{N}$.

i) Let $\left\{v_{\varepsilon}\right\}_{\varepsilon} \subset L^{p}(A)$ be a sequence converging to $v$ strongly in $L^{p}(A)$. Then, $\left\{v_{\varepsilon}\right\}_{\varepsilon}$ two-scale converges to the same limit $v$.

ii) Let $\left\{v_{\varepsilon}\right\}_{\varepsilon} \subset L^{p}(A)$ be a sequence two-scale converging to $v \in L^{p}(A \times Y)$. Then, $\left\{v_{\varepsilon}\right\}$ converges to $\int_{Y} v(\cdot, y) \mathrm{d} y$ weakly in $L^{p}(A)$.

iii) Let $\left\{v_{\varepsilon}\right\}_{\varepsilon}$ be a bounded sequence in $L^{p}(A)$. Then, there exist a subsequence of $\{\varepsilon\}$, still denoted by $\{\varepsilon\}$, and a function $v \in L^{p}(A \times Y)$ such that $\left\{v_{\varepsilon}\right\}_{\varepsilon}$ two-scale converges to $v$.

iv) Let $\left\{v_{\varepsilon}\right\}_{\varepsilon} \subset W^{1, p}(A)$ be a sequence such that $\left\{v_{\varepsilon}\right\}_{\varepsilon}$ and $\left\{\varepsilon D v_{\varepsilon}\right\}_{\varepsilon}$ are bounded in $L^{p}(A)$ and $\left(L^{p}(A)\right)^{N}$, respectively. Then, there exist a subsequence of $\{\varepsilon\}$, still denoted by $\{\varepsilon\}$, and a function $v \in L^{p}\left(A, W_{\text {per }}^{1, p}(Y)\right)$ such that $\left\{v_{\varepsilon}\right\}_{\varepsilon}$ and $\left\{\varepsilon D v_{\varepsilon}\right\}_{\varepsilon}$ two-scale converge to $v$ and $D_{y} v$, respectively.

Remark 2.3. Due to density properties, it is easily seen that if $\left\{v_{\varepsilon}\right\}_{\varepsilon} \subset L^{p}(A)$ two-scale converges to $v \in$ $L^{p}(A \times Y)$, convergence (2.1) holds true also for any function $\psi$ of the form $\psi(x, y)=\varphi_{1}(x) \varphi_{2}(y)$, where $\varphi_{1} \in C_{0}(A)$ and $\varphi_{2} \in L_{\mathrm{per}}^{\infty}(Y)$.

This section concludes with the following well-known Friedrichs inequality which will be an helpful tool to obtain a priori estimates for the solution of problem (1.7).

Proposition 2.4. Let $A$ be an open bounded connected subset of $\mathbb{R}^{N}$ with Lipschitz boundary and let $\Gamma \subset \partial A$ be such that the $(N-1)$-dimensional Hausdorff measure of $\Gamma$ is positive. Then, there exists a constant $c$ such that

$$
\int_{A}|v|^{p} \mathrm{~d} x \leq c\left(\int_{\Gamma}|v|^{p} \mathrm{~d} \sigma+\int_{A}|D v|^{p} \mathrm{~d} x\right) \quad \forall v \in W^{1, p}(A) .
$$

\section{A PRIORI NORM-ESTIMATES AND A CONVERGENCE RESUlT}

This section is devoted to prove convergences $(1.12,1.13)$ and (1.14). In Proposition 3.1, some a priori norm-estimates for $u_{\varepsilon}$, coming directly from equation (1.7), are given. The first difficulty is to obtain a priori $L^{p}\left(\Omega^{-}\right)$-norm estimate for the solution $u_{\varepsilon}$, independently of $\varepsilon$. These estimates are obtained in Proposition 3.3 by making use of the Friedrichs inequality. The second difficulty is to identify the weak limit of $\left\{u_{\varepsilon}\right\}_{\varepsilon}$ in $L^{p}\left(\Omega^{-}\right)$ as the trace on $\Sigma$ (the surface which separates $\Omega^{-}$and $\Omega^{+}$) of the weak limit of $\left\{\widetilde{u_{\varepsilon}}\right\}_{\varepsilon}$ in $V^{p}\left(\Omega^{+}\right)$. This result is proved in Proposition 3.4 by making use of the two-scale convergence method. 
Proposition 3.1. Let $u_{\varepsilon}$ be the unique solution of problem (1.7) under assumptions (1.4-1.6) and (1.10). Then, there exists a constant c such that

$$
\begin{gathered}
\left\|u_{\varepsilon}\right\|_{W^{1, p}\left(\Omega_{\varepsilon}^{+}\right)} \leq c \\
\left\|a\left(D u_{\varepsilon}\right)\right\|_{\left(L^{\frac{p}{p-1}}\left(\Omega_{\varepsilon}^{+}\right)\right)^{N} \leq c} \leq \\
\left\|h_{\varepsilon}^{\frac{1}{p}} u_{\varepsilon}\right\|_{L^{p}\left(\Omega^{-}\right)} \leq c \\
\left\|h_{\varepsilon}^{\frac{1}{p}}\left(D_{x^{\prime}} u_{\varepsilon}, \frac{1}{h_{\varepsilon}} \frac{\partial u_{\varepsilon}}{\partial x_{N}}\right)\right\|_{\left(L^{p}\left(\Omega^{-}\right)\right)^{N}} \leq c \\
\left\|h_{\varepsilon}^{\frac{p-1}{p}} a\left(D_{x^{\prime}} u_{\varepsilon}, \frac{1}{h_{\varepsilon}} \frac{\partial u_{\varepsilon}}{\partial x_{N}}\right)\right\|_{\left(L^{\frac{p}{p-1}}\left(\Omega^{-}\right)\right)^{N}} \leq c
\end{gathered}
$$

for every $\varepsilon$.

Proof. The a priori norm-estimates are obtained by choosing $v=u_{\varepsilon}$ as test function in (1.7) and by making use of $(1.5,1.6,1.10)$ and the Young inequality.

Corollary 3.2. Let $u_{\varepsilon}$ be the unique solution of problem (1.7) under assumptions (1.4-1.6) and (1.10). Then, there exists a constant $c$ such that

$$
\left\|u_{\varepsilon}\right\|_{L^{p}\left(\Omega_{\varepsilon}^{+} \cap \Sigma\right)} \leq c
$$

for every $\varepsilon$.

Proof. The estimate (3.6) follows from estimate (3.1), if one observes that there exists a constant $c$ such that

$$
\left\|u_{\varepsilon}\right\|_{L^{p}\left(\Omega_{\varepsilon}^{+} \cap \Sigma\right)}^{p} \leq c\left(\left\|u_{\varepsilon}\right\|_{L^{p}\left(\Omega_{\varepsilon}^{+}\right)}^{p}+\left\|\frac{\partial u_{\varepsilon}}{\partial x_{N}}\right\|_{L^{p}\left(\Omega_{\varepsilon}^{+}\right)}^{p}\right)
$$

for every $\varepsilon$.

Proposition 3.3. Let $u_{\varepsilon}$ be the unique solution of problem (1.7) under assumptions (1.4-1.6, 1.9) and (1.10). Then, there exists a constant c such that

$$
\left\|u_{\varepsilon}\right\|_{L^{p}\left(\Omega^{-}\right)} \leq c
$$

for every $\varepsilon$.

Proof. For the sake of clarity, first we prove (3.7) by assuming

$$
\left.\Omega^{-}=\bigcup_{k \in J_{\varepsilon}}(\varepsilon] 0,1\left[^{N-1}+\varepsilon k\right) \times\right]-1,0[
$$

for every $\varepsilon$, where $J_{\varepsilon}$ is defined in (1.1). Then, we sketch the proof of (3.7) for the general case.

By making use of the change of variable $x^{\prime}=\varepsilon y^{\prime}+\varepsilon k$ with $\left.y^{\prime} \in\right] 0,1\left[{ }^{N-1}\right.$, it results

$$
\int_{\Omega^{-}}\left|u_{\varepsilon}\right|^{p} \mathrm{~d} x=\sum_{k \in J_{\varepsilon}} \int_{\left.(\varepsilon] 0,1\left[N^{N-1}+\varepsilon k\right) \times\right]-1,0[}\left|u_{\varepsilon}\right|^{p} \mathrm{~d}\left(x^{\prime}, x_{N}\right)=\varepsilon^{N-1} \sum_{k \in J_{\varepsilon}} \int_{]_{0,1\left[{ }^{N-1} \times\right]-1,0[}}\left|u_{\varepsilon}\left(\varepsilon y^{\prime}+\varepsilon k, x_{N}\right)\right|^{p} \mathrm{~d}\left(y^{\prime}, x_{N}\right) .
$$


Then, the Friedrichs inequality (2.2) yields

$$
\begin{aligned}
\int_{\Omega^{-}}\left|u_{\varepsilon}\right|^{p} \mathrm{~d} x \leq & c \varepsilon^{N-1} \sum_{k \in J_{\varepsilon}}\left(\int_{\omega}\left|u_{\varepsilon}\left(\varepsilon y^{\prime}+\varepsilon k, 0\right)\right|^{p} \mathrm{~d} y^{\prime}+\varepsilon^{p} \int_{] 0,1\left[^{N-1} \times\right]-1,0[}\left|\left(D_{x^{\prime}} u_{\varepsilon}\right)\left(\varepsilon y^{\prime}+\varepsilon k, x_{N}\right)\right|^{p} \mathrm{~d}\left(y^{\prime}, x_{N}\right)\right. \\
& \left.\left.+\int_{] 0,1\left[^{N-1} \times\right]-1,0[} \mid \frac{\partial u_{\varepsilon}}{\partial x_{N}}\left(\varepsilon y^{\prime}+\varepsilon k, x_{N}\right)\right)\left.\right|^{p} \mathrm{~d}\left(y^{\prime}, x_{N}\right)\right) \\
= & c\left(\int_{\Omega_{\varepsilon}^{+} \cap \Sigma}\left|u_{\varepsilon}\right|^{p} \mathrm{~d} x^{\prime}+\varepsilon^{p} \int_{\Omega^{-}}\left|D_{x^{\prime}} u_{\varepsilon}\right|^{p} \mathrm{~d} x+\int_{\Omega^{-}}\left|\frac{\partial u_{\varepsilon}}{\partial x_{N}}\right|^{p} \mathrm{~d} x\right)
\end{aligned}
$$

for every $\varepsilon$, where the constant $c$, given in $(2.2)$, is independent of $\varepsilon$ and $u_{\varepsilon}(\cdot, 0)$ denotes the trace of $u_{\varepsilon}$ on $\Omega_{\varepsilon}^{+} \cap \Sigma$. Consequently, by making use of estimates (3.4) and (3.6), it follows that

$$
\int_{\Omega^{-}}\left|u_{\varepsilon}\right|^{p} \mathrm{~d} x \leq c\left(1+\frac{\varepsilon^{p}}{h_{\varepsilon}}+h_{\varepsilon}^{p-1}\right)
$$

for every $\varepsilon$, where $c$ is a constant independent of $\varepsilon$. Finally, estimate (3.7) is obtained using assumption (1.9).

Now, let us consider the general case. Let $J_{\varepsilon}^{\prime}=\left\{k \in \mathbb{N}^{N-1}:(\varepsilon] 0,1\left[^{N-1}+\varepsilon k\right) \cap(] 0, l_{1}[\times \cdots \times] 0, l_{N-1}[) \neq\right.$ $\left.\emptyset\}, C_{\varepsilon}=\bigcup_{k \in J_{\varepsilon}^{\prime}}(\varepsilon] 0,1\left[^{N-1}+\varepsilon k\right) \times\right]-1,0\left[, B \subset \mathbb{R}^{N-1}\right.$ be a bounded open set such that $\left.\bigcup_{k \in J_{\varepsilon}^{\prime}} \varepsilon\right] 0,1\left[^{N-1}+\varepsilon k \subset \subset\right.$ $B$ for every $\varepsilon$ and $C=B \times]-1,0[$. It is easy to prove the existence of a linear extension-operator $Q \in$ $L\left(W^{1, p}\left(\Omega^{-}\right), W^{1, p}(C)\right)$ such that (compare, for instance [6] and [10])

$$
\left\|D_{x^{\prime}} Q v\right\|_{\left(L^{p}(C)\right)^{N-1}} \leq c\left\|D_{x^{\prime}} v\right\|_{\left(L^{p}\left(\Omega^{-}\right)\right)^{N-1}}, \quad\left\|\frac{\partial Q v}{\partial x_{N}}\right\|_{L^{p}(C)} \leq c\left\|\frac{\partial v}{\partial x_{N}}\right\|_{L^{p}\left(\Omega^{-}\right)} \quad \forall v \in W^{1, p}\left(\Omega^{-}\right),
$$

where $c$ is a constant independent of $v$. In particular, we have, on one hand,

$$
\int_{\Omega^{-}}\left|u_{\varepsilon}\right|^{p} \mathrm{~d} x \leq \int_{C_{\varepsilon}}\left|Q u_{\varepsilon}\right|^{p} \mathrm{~d} x
$$

for every $\varepsilon$. On the other hand, by an argument similar to that used in the proof of (3.8), one can prove that

$$
\int_{C_{\varepsilon}}\left|Q u_{\varepsilon}\right|^{p} \mathrm{~d} x \leq c\left(\int_{\Omega_{\varepsilon}^{+} \cap \Sigma}\left|u_{\varepsilon}\right|^{p} \mathrm{~d} x^{\prime}+\varepsilon^{p} \int_{C_{\varepsilon}}\left|D_{x^{\prime}} Q u_{\varepsilon}\right|^{p} \mathrm{~d} x+\int_{C_{\varepsilon}}\left|\frac{\partial Q u_{\varepsilon}}{\partial x_{N}}\right|^{p} \mathrm{~d} x\right),
$$

for every $\varepsilon$, where $c$ is a constant dependent on $N$, but independent of $\varepsilon$. Indeed, the proof of (3.8) would rather lead to $\int_{\cup_{k \in J_{\varepsilon}^{\prime}(\varepsilon \omega+\varepsilon k)}}\left|Q u_{\varepsilon}\right|^{p} \mathrm{~d} x^{\prime}$ as first term in the right hand-side of (3.12). To obtain the smaller quantity $\int_{\Omega_{\varepsilon}^{+} \cap \Sigma}\left|u_{\varepsilon}\right|^{p} \mathrm{~d} x^{\prime}$, one has to use the definition of $C_{\varepsilon}$. This definition allows, upon joining up a fixed number (depending only on $N$ ) of cells of $C_{\varepsilon}$, to control the $L^{p}$-norm of $Q u_{\varepsilon}$ on the cells of $C_{\varepsilon}$ crossed by the lateral boundary of $\Omega^{-}$, only in terms of the $L^{p}$-norm of $\varepsilon D_{x^{\prime}} Q u_{\varepsilon}$ and $\frac{\partial Q u_{\varepsilon}}{\partial x_{N}}$ on the same cells, plus the $L^{p}$-norm of the trace of $u_{\varepsilon}$ on a part of the upper surface of cells of $C_{\varepsilon}$ completely included in $\Omega^{-}$.

By combining (3.11) and (3.12) with (3.10), it follows that

$$
\int_{\Omega^{-}}\left|u_{\varepsilon}\right|^{p} \mathrm{~d} x \leq c\left(\int_{\Omega_{\varepsilon}^{+} \cap \Sigma}\left|u_{\varepsilon}\right|^{p} \mathrm{~d} x^{\prime}+\varepsilon^{p} \int_{\Omega^{-}}\left|D_{x^{\prime}} u_{\varepsilon}\right|^{p} \mathrm{~d} x+\int_{\Omega^{-}}\left|\frac{\partial u_{\varepsilon}}{\partial x_{N}}\right|^{p} \mathrm{~d} x\right)
$$

for every $\varepsilon$, where $c$ is a constant independent of $\varepsilon$. Finally, by making use of $(3.4,3.6)$ and (1.9), we obtain (3.7). 
Proposition 3.4. Let $u_{\varepsilon}$ be the unique solution of problem (1.7) under assumptions (1.4-1.6) and (1.10), let $|\omega|$ be the constant given by (1.2) and let $V^{p}\left(\Omega^{+}\right)$be the space defined in (1.11). Then (1.14) holds true. Moreover, there exist a subsequence of $\{\varepsilon\}$, still denoted by $\{\varepsilon\}$, and $u \in V^{p}\left(\Omega^{+}\right)$such that (1.12) holds true. Furthermore, for this subsequence, under assumption (1.9), convergence (1.13) holds true.

Remark 3.5. In the spirit of Remark 1.3, let us point out that convergences (1.12) and (1.14) hold true without assumption (1.9).

Proof of Proposition 3.4. Convergence (1.14) follows from (3.4). By virtue of (3.1), there exist a subsequence of $\{\varepsilon\}$, still denoted by $\{\varepsilon\}$, and $u \in V^{p}\left(\Omega^{+}\right)$such that (1.12) holds true (see [5,6] and [11]). Moreover, under assumption (1.9), estimate (3.7) holds true. Consequently, in view of (1.14), there exist a subsequence of the previous one, still denoted by $\{\varepsilon\}$, and $w \in L^{p}\left(\Omega^{-}\right)$, independent of $x_{N}$, such that

$$
u_{\varepsilon} \rightarrow w \text { weakly in } L^{p}\left(\Omega^{-}\right) .
$$

In order to prove (1.13), we show that, up to a subsequence,

$$
\left\{u_{\varepsilon}\right\}_{\varepsilon} \text { two-scale converges to } w \text { in } \Omega^{-},
$$

too. To this aim, first observe that (3.4) and (1.9) provide that $\lim _{\varepsilon \rightarrow 0}\left\|\varepsilon D u_{\varepsilon}\right\|_{\left(L^{p}\left(\Omega^{-}\right)\right)^{N}}=0$ Consequently, by making use of Proposition 2.2-i, it follows that

$$
\left\{\varepsilon D u_{\varepsilon}\right\}_{\varepsilon} \text { two-scale converges to }(0, \cdots, 0) \text { in } \Omega^{-} .
$$

By combining (3.7) with (3.15), by virtue of Proposition 2.2-iv, there exist a subsequence of the previous selected one, still denoted by $\{\varepsilon\}$, and $g \in L^{p}\left(\Omega^{-}\right)$(independent of $\left.y \in[0,1]^{N} !\right)$ such that

$$
\left\{u_{\varepsilon}\right\}_{\varepsilon} \text { two-scale converges to } g \text { in } \Omega^{-} \text {. }
$$

Finally, since $g$ is independent of $y,(3.14)$ follows by comparing (3.13) with (3.16) and by making use of Proposition 2.2-ii.

Let us point out that the two-scale convergence allows us to pass to the limit in the product of weak convergences. In fact, by setting $\psi_{\varepsilon}(x)=\chi_{\Omega_{\varepsilon}^{+} \cap \Sigma}\left(x^{\prime}\right)$ for $x=\left(x^{\prime}, x_{N}\right) \in \Omega^{-}$, where $\chi_{\Omega_{\varepsilon}^{+} \cap \Sigma}$ denotes the characteristic function of $\Omega_{\varepsilon}^{+} \cap \Sigma$ in $\Sigma$, convergence (3.14) provides that (see Def. 2.1 and Rem. 2.3)

$$
\lim _{\varepsilon} \int_{\Omega^{-}} u_{\varepsilon} \psi_{\varepsilon} \varphi \mathrm{d} x=|\omega| \int_{\Omega^{-}} w \varphi \mathrm{d} x \quad \forall \varphi \in C_{0}\left(\Omega^{-}\right),
$$

from which it follows that, since $\left\{u_{\varepsilon} \psi_{\varepsilon}\right\}_{\varepsilon}$ is bounded in $L^{p}\left(\Omega^{-}\right)$,

$$
u_{\varepsilon} \psi_{\varepsilon} \rightarrow|\omega| w \text { weakly in } L^{p}\left(\Omega^{-}\right) .
$$

Moreover, equation (1.14) provides that

$$
\lim _{\varepsilon}\left\|\frac{\partial\left(u_{\varepsilon} \psi_{\varepsilon}\right)}{\partial x_{N}}\right\|_{L^{p}\left(\Omega^{-}\right)}=\lim _{\varepsilon}\left\|\psi_{\varepsilon} \frac{\partial u_{\varepsilon}}{\partial x_{N}}\right\|_{L^{p}\left(\Omega^{-}\right)} \leq \lim _{\varepsilon}\left\|\frac{\partial u_{\varepsilon}}{\partial x_{N}}\right\|_{L^{p}\left(\Omega^{-}\right)}=0 .
$$

Then, by making use of (3.17) and (3.18), and by recalling that $w$ is independent of $x_{N}$, it results that

$$
\begin{aligned}
\lim _{\varepsilon} \int_{\Sigma} \widetilde{u_{\varepsilon}} \varphi \mathrm{d} x^{\prime} & =\lim _{\varepsilon}\left(\int_{\Omega^{-}} \frac{\partial\left(u_{\varepsilon} \psi_{\varepsilon}\right)}{\partial x_{N}} \varphi \mathrm{d} x+\int_{\Omega^{-}} u_{\varepsilon} \psi_{\varepsilon} \frac{\partial \varphi}{\partial x_{N}} \mathrm{~d} x\right) \\
& =|\omega| \int_{\Omega^{-}} w \frac{\partial \varphi}{\partial x_{N}} \mathrm{~d} x=|\omega| \int_{\Sigma} w \varphi \mathrm{d} x^{\prime} \quad \forall \varphi \in C_{0}^{\infty}\left(\Omega^{+} \cup \Sigma \cup \Omega^{-}\right) .
\end{aligned}
$$


On the other hand, from (1.12) it follows that

$$
\begin{aligned}
\lim _{\varepsilon} \int_{\Sigma} \widetilde{u_{\varepsilon}} \varphi \mathrm{d} x^{\prime} & =-\lim _{\varepsilon}\left(\int_{\Omega^{+}} \frac{\partial \widetilde{u_{\varepsilon}}}{\partial x_{N}} \varphi \mathrm{d} x+\int_{\Omega^{+}} \widetilde{u_{\varepsilon}} \frac{\partial \varphi}{\partial x_{N}} \mathrm{~d} x\right) \\
& =-|\omega| \int_{\Omega^{+}} \frac{\partial u}{\partial x_{N}} \varphi \mathrm{d} x-|\omega| \int_{\Omega^{+}} u \frac{\partial \varphi}{\partial x_{N}} \mathrm{~d} x=|\omega| \int_{\Sigma} u \varphi \mathrm{d} x^{\prime} \quad \forall \varphi \in C_{0}^{\infty}\left(\Omega^{+} \cup \Sigma \cup \Omega^{-}\right) .
\end{aligned}
$$

By comparing (3.19) with (3.20), we obtain

$$
\int_{\Sigma} w \varphi \mathrm{d} x^{\prime}=\int_{\Sigma} u \varphi \mathrm{d} x^{\prime} \quad \forall \varphi \in C_{0}^{\infty}(\Sigma)
$$

Then, $w$ is the function independent of $x_{N}$ which is equal, on $\Sigma$, to the trace of $u$. Finally, convergence (1.13) follows from (3.13).

Remark 3.6. In order to obtain (3.7), and consequently (3.13), we may only assume $\left\{\frac{\varepsilon^{p}}{h_{\varepsilon}}\right\}_{\varepsilon}$ bounded (see (3.9)), but in the proof of (3.15) we need $\lim _{\varepsilon \rightarrow 0} \frac{\varepsilon^{p}}{h_{\varepsilon}}=0$.

\section{Proof of Theorem 1.1}

We sketch the proofs of $(1.12,1.15,1.16)$ and $(1.17)$ which are similar (except for the last one) to the corresponding ones in Theorem 1.2 in [5].

By virtue of $(3.1,3.2)$ and Proposition 3.4, there exist a subsequence of $\{\varepsilon\}$, still denoted by $\{\varepsilon\}$, and $u \in V^{p}\left(\Omega^{+}\right), d=\left(d_{1}, \cdots, d_{N-1}\right) \in\left(L^{p}\left(\Omega^{+}\right)\right)^{N-1}, z \in L^{\frac{p}{p-1}}\left(\Omega^{+}\right)$and $\eta=\left(\eta_{1}, \cdots, \eta_{N}\right) \in\left(L^{\frac{p}{p-1}}\left(\Omega^{+}\right)\right)^{N}$ satisfying $(1.12,1.15)$ and

$$
\left|\widetilde{u_{\varepsilon}}\right|^{p-2} \widetilde{u_{\varepsilon}} \rightarrow z \text { weakly in } L^{\frac{p}{p-1}}\left(\Omega^{+}\right), \quad\left[a\left(D u_{\varepsilon}\right)\right]^{\sim} \rightarrow \eta \text { weakly in }\left(L^{\frac{p}{p-1}}\left(\Omega^{+}\right)\right)^{N} .
$$

By arguing as in [5] (p. 1064), it results that

$$
\eta_{i}=0 \quad \text { a.e. in } \Omega^{+}, \quad \forall i \in\{1, \cdots, N-1\} .
$$

Now, let us prove the convergence of the energies. For any $v \in C^{1}\left(\overline{\Omega^{+}}\right)$, let us pass to the limit in (1.7), as $\varepsilon \rightarrow 0$, with the test function:

$$
w(x)=\left\{\begin{array}{l}
v(x) \text { if } x \in \Omega^{+} \\
v\left(x^{\prime}, 0\right) \text { if } x \in \Omega^{-}
\end{array} \in W^{1, p}\left(\Omega^{+} \cup \Omega^{-}\right) .\right.
$$

Then, by virtue of $(1.2,1.10,3.3,3.5,4.1)$ and $(4.2)$, one has that

$$
\int_{\Omega^{+}} \eta_{N} \frac{\partial v}{\partial x_{N}}+z v \mathrm{~d} x=|\omega| \int_{\Omega^{+}} f v \mathrm{~d} x \quad \forall v \in C^{1}\left(\overline{\Omega^{+}}\right),
$$

i.e., by density argument (compare Prop. 4.1 in [11]),

$$
\int_{\Omega^{+}} \eta_{N} \frac{\partial v}{\partial x_{N}}+z v \mathrm{~d} x=|\omega| \int_{\Omega^{+}} f v \mathrm{~d} x \quad \forall v \in V\left(\Omega^{+}\right) .
$$


In particular, (4.3) holds true for $v=u$. Consequently, by choosing $v=u_{\varepsilon}$ as test function in (1.7), by virtue of $(1.10,1.12)$ and $(3.3)$ one obtain the convergence of the energies:

$$
\begin{array}{r}
\lim _{\varepsilon \rightarrow 0}\left(\int_{\Omega_{\varepsilon}^{+}} a\left(D u_{\varepsilon}\right) D u_{\varepsilon}+\left|u_{\varepsilon}\right|^{p} \mathrm{~d} x+h_{\varepsilon} \int_{\Omega^{-}} a\left(D_{x^{\prime}} u_{\varepsilon}, \frac{1}{h_{\varepsilon}} \frac{\partial u_{\varepsilon}}{\partial x_{N}}\right)\right. \\
\left.\left(D_{x^{\prime}} u_{\varepsilon}, \frac{1}{h_{\varepsilon}} \frac{\partial u_{\varepsilon}}{\partial x_{N}}\right)+\left|u_{\varepsilon}\right|^{p} \mathrm{~d} x\right) \\
=|\omega| \int_{\Omega^{+}} f u \mathrm{~d} x=\int_{\Omega^{+}} \eta_{N} \frac{\partial u}{\partial x_{N}}+z u \mathrm{~d} x .
\end{array}
$$

As in [5] (p. 1065) $(1.2,1.12,1.15,4.1,4.2)$ and (4.4) allow us to obtain the following monotone relation:

$$
\begin{aligned}
\frac{1}{t} \int_{\Omega^{+}} \eta_{N}\left(\frac{\partial u}{\partial x_{N}}-\tau_{N}\right) & -a(\tau)\left(\left(d,|\omega| \frac{\partial u}{\partial x_{N}}\right)-|\omega| \tau\right) \mathrm{d} x \\
& \left.+\frac{1}{t} \int_{\Omega^{+}}\left(z-|\omega||v|^{p-2} v\right)(u-v) \mathrm{d} x \geq 0 \forall \tau \in\left(L^{p}\left(\Omega^{+}\right)\right)^{n}, \quad \forall v \in L^{p}\left(\Omega^{+}\right), \forall t \in\right] 0,+\infty[,
\end{aligned}
$$

which will enable us to derive (see again in [5], pp. 1065-1068)

$$
\begin{gathered}
z=|\omega||u|^{p-2} u, \quad \eta_{N}=|\omega| a_{N}\left(\frac{d_{1}}{|\omega|}, \cdots, \frac{d_{N-1}}{|\omega|}, \frac{\partial u}{\partial x_{N}}\right) \quad \text { a.e. in } \Omega^{+}, \\
a_{i}\left(\frac{d_{1}}{|\omega|}, \cdots, \frac{d_{N-1}}{|\omega|}, \frac{\partial u}{\partial x_{N}}\right)=0 \quad \text { a.e. in } \Omega^{+}, \quad \forall i \in\{1, \cdots, N-1\} .
\end{gathered}
$$

By combining (4.3) with (4.5) and (4.6), it results that $\left(u, d_{1}, \cdots, d_{N-1}\right)$ is a weak solution of problem (1.16). Since this problem admits a unique solution $u$ (see [5], pp. 1068-1069), convergences (1.12) holds true for the whole sequence $\left\{u_{\varepsilon}\right\}_{\varepsilon}$. The convergence of the energies (1.17) follows from (4.4) and (4.5). If $a$ is strictly monotone, the uniqueness of the solution $\left(u, d_{1}, \cdots, d_{N-1}\right) \in V^{p}\left(\Omega^{+}\right) \times\left(L^{p}\left(\Omega^{+}\right)\right)^{N-1}$ of problem (1.16) is proved in [5] (pp. 1068-1069). Consequently, in this case, convergence (1.15) holds true for the whole sequence $\left\{u_{\varepsilon}\right\}_{\varepsilon}$.

Convergences (1.13) and (1.14) are proved in Proposition 3.4, under assumption (1.9) for the first one.

Remark 4.1. If in $(1.3)$ we replace $-\operatorname{div}\left(a\left(D U_{\varepsilon}\right)\right)$ with $-\operatorname{div}\left(a\left(x, D U_{\varepsilon}\right)\right)$ in $\Omega_{\varepsilon}^{+}$and $-\operatorname{div}\left(a\left(x^{\prime}, \frac{x_{N}}{h_{\varepsilon}}, D U_{\varepsilon}\right)\right)$ in $\Omega_{\varepsilon}^{-}$, where $a(x, \xi)$ is a Carathéodory function satisfying usual monotonicity, coercivity and growth conditions, it is evident that Theorem 1.1 and Corollary 1.2 hold true with $a$ depending also on $x$. Similarly, the results obtained in the present paper are still valid if the local nonlinearity $\left|U_{\varepsilon}\right|^{p-2} U_{\varepsilon}$ is replaced by $b\left(x, U_{\varepsilon}\right)$ in $\Omega_{\varepsilon}^{+}$and $b\left(x^{\prime}, \frac{x_{N}}{h_{\varepsilon}}, U_{\varepsilon}\right)$ in $\Omega_{\varepsilon}^{-}$, where $b(x, s)$ is a Carathéodory function, strictly monotone with respect to $s$ and with $p-1$ growth at infinity.

The authors are grateful to V.V. Jikov for helpful discussions. This work is partially supported by the C.N.R. Project: "Agenzia 2000"

\section{REFERENCES}

[1] G. Allaire, Homogenization and Two-Scale Convergence. SIAM J. Math Anal. 23 (1992) 1482-1518.

[2] G. Allaire and M. Amar, Boundary Layer Tails in Periodic Homogenization. ESAIM: COCV 4 (1999) $209-243$.

[3] Y. Amirat and O. Bodart, Boundary Layer Correctors for the Solution of Laplace Equation in a Domain with Oscillating Boundary. J. Anal. Appl. 20 (2001) 929-940.

[4] N. Ansini and A. Braides, Homogenization of Oscillating Boundaries and Applications to Thin Films. J. Anal. Math. 83 (2001) $151-183$.

[5] D. Blanchard, L. Carbone and A. Gaudiello, Homogenization of a Monotone Problem in a Domain with Oscillating Boundary. ESAIM: M2AN 33 (1999) 1057-1070. 
[6] R. Brizzi and J.P. Chalot, Boundary Homogenization and Neumann Boundary Value Problem. Ricerche Mat. 46 (1997) 341-387.

[7] G. Buttazzo and R.V. Kohn, Reinforcement by a Thin Layer with Oscillating Thickness. Appl. Math. Optim. 16 (1987) 247-261.

[8] G.A. Chechkin, A. Friedman and A.L. Piatniski, The Boundary Value Problem in a Domain with Very Rapidly Oscillating Boundary. J. Math. Anal. Appl. 231 (1999) 213-234.

[9] P.G. Ciarlet and P. Destuynder, A Justification of the Two-Dimensional Linear Plate Model. J. Mécanique 18 (1979) 315-344.

[10] D. Cioranescu and J. Saint Jean Paulin, Homogenization in Open Sets with Holes. J. Math. Anal. Appl. 71 (1979) $590-607$.

[11] A. Corbo Esposito, P. Donato, A. Gaudiello and C. Picard, Homogenization of the $p$-Laplacian in a Domain with Oscillating Boundary. Comm. Appl. Nonlinear Anal. 4 (1997) 1-23.

[12] A. Gaudiello, Asymptotic Behaviour of non-Homogeneous Neumann Problems in Domains with Oscillating Boundary. Ricerche Mat. 43 (1994) 239-292.

[13] A. Gaudiello, Homogenization of an Elliptic Transmission Problem. Adv. Math Sci. Appl. 5 (1995) 639-657.

[14] A. Gaudiello, B. Gustafsson, C. Lefter and J. Mossino, Asymptotic Analysis for Monotone Quasilinear Problems in Thin Multidomains. Differential Integral Equations 15 (2002) 623-640.

[15] A. Gaudiello, R. Hadiji and C. Picard, Homogenization of the Ginzburg-Landau Equation in a Domain with Oscillating Boundary. Commun. Appl. Anal. (to appear).

[16] A. Gaudiello, R. Monneau, J. Mossino, F. Murat and A. Sili, On the Junction of Elastic Plates and Beams. C. R. Acad. Sci. Paris Sér. I 335 (2002) 717-722.

[17] H. Le Dret, Problèmes variationnels dans les multi-domaines : modélisation des jonctions et applications. Masson, Paris (1991)

[18] J.L. Lions, Quelques méthodes de résolution de problèmes aux limites non linéaires. Dunod, Paris (1969).

[19] T.A. Mel'nyk, Homogenization of the Poisson Equations in a Thick Periodic Junction. ZAA J. Anal. Appl. 18 (1999) 953-975.

[20] T.A. Mel'nyk and S.A. Nazarov, Asymptotics of the Neumann Spectral Problem Solution in a Domain of "Thick Comb" Type. J. Math. Sci. 85 (1997) 2326-2346.

[21] G. Nguetseng, A General Convergence Result for a Functional Related to the Theory of Homogenization. SIAM J. Math Anal. 20 (1989) 608-623.

[22] L. Tartar, Cours Peccot, Collège de France (March 1977). Partially written in F. Murat, H-Convergence, Séminaire d'analyse fonctionnelle et numérique de l'Université d'Alger (1977-78). English translation in Mathematical Modelling of Composite Materials, edited by A. Cherkaev and R.V. Kohn, Progress in Nonlinear Differential Equations and their Applications, BirkhäuserVerlag (1997) 21-44. 